\title{
Numerical Simulation of a Steady-State Electron Shock Wave in a Submicrometer Semiconductor Device
}

\author{
Carl L. Gardner
}

\begin{abstract}
The hydrodynamic model consists of a set of nonlinear conservation laws for particle number, momentum, and energy, coupled to Poisson's equation for the electric potential. The nonlinear conservation laws are just the Euler equations of gas dynamics for a gas of charged particles in an electric field, with the addition of a heat conduction term. Thus the hydrodynamic model PDE's have hyperbolic, parabolic, and elliptic modes.

The nonlinear hyperbolic modes support shock waves. The first numerical simulations of a steady-state electron shock wave in a semiconductor device are presented, using the hydrodynamic model. For the ballistic diode (which models the channel of a MOSFET), the shock wave is fully developed in $\mathrm{Si}$ (with $1-\mathrm{V}$ bias) at $300 \mathrm{~K}$ for a $0.1-\mu \mathrm{m}$ channel and at $77 \mathrm{~K}$ for a $1.0-\mu \mathrm{m}$ channel.
\end{abstract}

\section{INTRODUCTION}

$\mathrm{T}_{\mathrm{in}}$ HE hydrodynamic model treats the propagation of electrons in a semiconductor device as the flow of a compressible charged fluid. This electron gas has a soundspeed, ${ }^{1}$ and the electron flow may be either subsonic or supersonic. In the case of supersonic flow, electron shock waves will in general develop in semiconductor devices.

The hydrodynamic equations consist of a set of nonlinear conservation laws for particle number, momentum, and energy, coupled to Poisson's equation for the electric potential. The nonlinear conservation laws are just the Euler equations of gas dynamics ${ }^{2}$ for a gas of charged particles in an electric field, with the addition of a heat conduction term. Thus the hydrodynamic model PDE's have hyperbolic, parabolic, and elliptic modes.

The nonlinear hyperbolic modes support nonlinear shock and contact waves. Electron shock waves should occur in silicon devices at short length scales or at low temperatures. The first numerical simulations of a steady-state electron shock wave in a semiconductor device will be presented, using the hydrodynamic model. The simulations were performed for the ballistic diode (which models the channel of a MOSFET). With a $1-\mathrm{V}$ bias across the diode, the shock wave is fully developed in $\mathrm{Si}$ at $300 \mathrm{~K}$ for a $0.1-\mu \mathrm{m}$ channel and at $77 \mathrm{~K}$ for a $1.0-\mu \mathrm{m}$ channel.

This investigation focuses on appropriate numerical methods for steady-state simulations (including shock waves) when the

Manuscript received March 21, 1990; revised September 17, 1990. This research was supported in part by the National Science Foundation under Grant DMS-8905872. The review of this paper was arranged by Associate Editor S. E. Laux.

The author is with the Department of Computer Science, Duke University, Durham, NC 27706

IEEE Log Number 9040825.

'The soundspeed in the electron gas is unrelated to the soundspeed in the phonon gas.

${ }^{2}$ For an introduction to the Euler equations and shock waves see [1] electron flow is both subsonic and supersonic. The one-dimensional (ID) steady-state hydrodynamic equations will then be elliptic in the subsonic regions and hyperbolic/elliptic in the supersonic regions. Central differences may be used in the elliptic regions. However, in the hyperbolic/elliptic regions, central differences are unstable and a hyperbolic method (e.g., some form of upwinding) must be used. In this paper, I will use a second upwind method for both elliptic and hyperbolic/elliptic regions. In the elliptic regions, the second upwind method is related to the Scharfetter-Gummel exponential fitting method.

I give a brief introduction to the hydrodynamic model in Section II, and describe the nonlinear waves that the model supports. In Section III, I describe the ballistic diode problem, and discuss the central difference and second upwind discretizations of the 1D steady-state hydrodynamic equations. In Section IV I compare central difference and second upwind computations for steady-state subsonic flow of electrons in the ballistic diode. In Section V, I discuss transonic electron flow in the ballistic diode using the second upwind method. Section V-A presents a steady-state Mach 1.6 electron shock wave in $\mathrm{Si}$ at $300 \mathrm{~K}$, and Section V-B presents a steady-state Mach 4.1 electron shock wave in $\mathrm{Si}$ at $77 \mathrm{~K}$. These electron shock waves are analogous to the shock wave that forms in a converging-diverging Laval nozzle for transonic gas flow. In Section VI, I make some concluding remarks.

\section{THE HydrodynamiC MOdel}

The hydrodynamic equations are

$$
\begin{gathered}
\frac{\partial n}{\partial t}+\nabla \cdot(n \boldsymbol{v})=\left(\frac{\partial n}{\partial t}\right)_{c} \\
\frac{\partial \boldsymbol{p}}{\partial t}+\boldsymbol{v} \nabla \cdot \boldsymbol{p}+\boldsymbol{p} \cdot \nabla \boldsymbol{v}=-e n \boldsymbol{E}-\nabla(n T)+\left(\frac{\partial p}{\partial t}\right)_{c} \\
\frac{\partial W}{\partial t}+\nabla \cdot(\boldsymbol{v} W)=-e n \boldsymbol{v} \cdot \boldsymbol{E}-\nabla \cdot(\boldsymbol{v} n T) \\
+\nabla \cdot(\kappa \nabla T)+\left(\frac{\partial W}{\partial t}\right)_{c} \\
\nabla \cdot(\epsilon \nabla \phi)=-e\left(N_{D}-N_{A}-n\right), \quad E=-\nabla \phi
\end{gathered}
$$

where $n$ is the electron density, $v$ is the velocity, $p$ is the momentum density, $e(>0)$ is the electronic charge, $\boldsymbol{E}$ is the electric field, $T$ is the temperature in energy units (Boltzmann's constant $k$ has been set to 1 ), $W$ is the energy density, $\kappa$ is the 
thermal conductivity, the subscript $c$ indicates collision ${ }^{3}$ terms, $\epsilon$ is the dielectric constant, $\phi$ is the electric potential, $N_{D}$ is the density of donors, and $N_{A}$ is the density of acceptors. Equation (1) expresses conservation of electron number, (2) expresses conservation of momentum, (3) expresses conservation of energy, and (4) is Poisson's equation for the electric potential.

The transport equations (1)-(3) were derived by Blødtekjær [2] as the first three moments of the Boltzmann equation.

The hydrodynamic model can also describe multiple species of charge carriers (e.g., upper and lower valley electrons and holes) by adding extra copies of (1)-(3).

To close the set of equations, I will assume that the energy bands are parabolic

$$
p=m n v \quad W=\frac{3}{2} n T+\frac{1}{2} m n v^{2}
$$

where $m$ is the effective electron mass.

Equations (1)-(3) are in conservation form, and may be written in terms of the variables $n, v, T$, and $\phi$. These variables represent the simplest choice for upwind methods and for the ballistic diode boundary conditions. In addition, discretizing the PDE's is conservation form eliminates some of the discretization ambiguities that plague nonconservation forms of the PDE's, and convergence of the Newton method used to solve the steady-state equations is more robust.

In the absence of heat conduction ( $\kappa=0$ in (3)), (1)-(3) are the Euler equations with source terms due to the collision terms and the electric field. The polytropic gas constant $\gamma=\frac{5}{3}$ for the "monatomic" electron gas ( $\gamma$ represents the relationship between the total internal energy/particle $U=W / n-m v^{2} / 2$ of the gas and the temperature $T:(\gamma-1) U=T$. $)$ The transport equations then are hyperbolic, with five nonlinear waves [1], [3]:

\begin{tabular}{lcc}
\hline Vonlinear Waves & Characteristic Speed & Discontinuous Variables \\
\hline 2 shocks & $\lambda_{ \pm}=v \pm c$ & $v, n, T$ \\
3 contacts & $\lambda_{0}=v$ & $\boldsymbol{v}_{T}, T$ \\
\hline
\end{tabular}

Here $v$ is the particle velocity normal to the wave, and $\boldsymbol{v}_{T}$ is the particle velocity tangential to the wave. The soundspeed $c=$ $\sqrt{\gamma T / m}$.

With heat conduction (nonzero $\kappa$ ), (1)-(3) are hyperbolic (4 modes) plus parabolic ( 1 mode) [4]. There are now only four nonlinear hyperbolic waves

\begin{tabular}{lcc}
\hline Nonlinear Waves & Characteristic Speed & Discontinuous Variables \\
\hline 2 shocks & $\lambda_{ \pm}=v \pm c$ & $v, n$ \\
2 contacts & $\lambda_{0}=v$ & $v_{T}$ \\
\hline
\end{tabular}

The contact wave corresponding to a discontinuity in $T$ has disappeared due to the parabolic heat conduction term $\nabla \cdot(\kappa \nabla T)$ in (3), and the soundspeed $c=\sqrt{T / m}$. The limit $\kappa \rightarrow 0$ is a singular limit, with a discontinuous change in the soundspeed and the number of nonlinear waves (see [4] for a detailed analysis). For $T=\alpha T_{0}$ and $T_{0}=300 \mathrm{~K}, c=\sqrt{\alpha} 1.3 \times 10^{7} \mathrm{~cm} / \mathrm{s}$, while for $T_{0}=77 \mathrm{~K}, c=\sqrt{\alpha} 6.6 \times 10^{6} \mathrm{~cm} / \mathrm{s}$.

${ }^{3}$ The collision terms may include the effects of electron-phonon and electron-impurity collisions, electron-hole generation and recombination, intervalley and interband scattering, etc.

\section{The Ballistic Diode Problem}

The $n^{+}-n-n^{+}$ballistic diode models electron flow in the channel of a MOSFET, and exhibits hot electron effects at scales on the order of a micrometer. The diode begins with an $\mathrm{n}^{+}$ "source" region, is followed by an n "channel" region, and ends with an $\mathrm{n}^{+}$" "drain" region.

The effects of holes may be neglected for the ballistic diode problem. This implies that

$$
\left(\frac{\partial n}{\partial t}\right)_{c}=0 .
$$

The collision terms in (2) and (3) are approximated in terms of momentum and energy relaxation times. For the relaxation times and the thermal conductivity, I take the Baccarani-Wordeman models [5]

$$
\begin{aligned}
\left(\frac{\partial \boldsymbol{p}}{\partial t}\right)_{c} & =\frac{-\boldsymbol{p}}{\tau_{p}}, \quad \tau_{p}=m \frac{\mu_{n 0}}{e} \frac{T_{0}}{T} \\
\left(\frac{\partial W}{\partial t}\right)_{c} & =\frac{-\left(W-\frac{3}{2} n T_{0}\right)}{\tau_{w}} \\
\tau_{w} & =\frac{m}{2} \frac{\mu_{n 0}}{e} \frac{T_{0}}{T}+\frac{3}{2} \frac{\mu_{n 0}}{e v_{s}^{2}} \frac{T T_{0}}{T+T_{0}} \\
\kappa & =\kappa_{0} \frac{\mu_{n 0}}{e} n T_{0}
\end{aligned}
$$

where $T_{0}$ is the ambient device temperature, $\mu_{n 0}=\mu_{n 0}\left(T_{0}, N_{D}\right.$ $\left.+N_{A}\right)$ is the low-field electron mobility, and $v_{s}=v_{s}\left(T_{0}\right)$ is the saturation velocity. The models for $\tau_{p}$ and $\tau_{w}$ include the effects of electron-phonon and electron-impurity collisions.

In $1 \mathrm{D}$, the hydrodynamic model consists of three nonlinear conservation laws (for electron number, momentum, and energy), plus Poisson's equation for the electric potential

$$
\begin{aligned}
f_{n}= & \frac{d}{d \mathrm{x}}(n v)=0 \\
f_{v}= & \frac{d}{d v}\left(m n v^{2}\right)-e n \frac{d \phi}{d x}+\frac{d}{d x}(n T)+\frac{m n v}{\tau_{p}}=0 \\
f_{T}= & \frac{d}{d x}\left(\frac{5}{2} n v T+\frac{1}{2} m n v^{3}-e n v \phi\right) \\
& -\frac{d}{d x}\left(\kappa \frac{d T}{d x}\right)+\frac{\frac{1}{2} m n v^{2}+\frac{3}{2} n\left(T-T_{0}\right)}{\tau_{w}}=0 \\
f_{\phi}= & \epsilon \frac{d^{2} \phi}{d x^{2}}+e(N-n)=0
\end{aligned}
$$

For boundary conditions I take charge neutral contacts $(n=$ $N$ ) in thermal equilibrium ( $T=T_{0}$ ) with the ambient temperature at $x_{\min }$ and $x_{\max }$, with a bias $V$ across the device: $e \phi\left(x_{\min }\right)$ $=T \ln \left(n / n_{i}\right)$ and $e \phi\left(x_{\max }\right)=T \ln \left(n / n_{i}\right)+e V$, where $n_{i}$ is the intrinsic electron concentration.

The variables $n, T$, and $\phi$ are defined at the grid points $i=$ $0,1, \cdots, N-1, N$, while the velocity $v$ is defined at the midpoints of the elements $l_{i}(i=1, \cdots, N)$ connecting grid points $i-1$ and $i$. The boundary conditions specify $n, T$, and $\phi$ at $i=0$ and $i=N$. Equations (10), (12), and (13) are enforced at the interior grid points $i=1, \cdots, N-1$, while (11) is enforced at the midpoints of the elements $l_{i}, i=1, \cdots, N$. The discrete equations are then derived by using either central 
differences (if the flow is everywhere subsonic) or the second upwind method (for subsonic or transonic flow problems).

The second upwind method ${ }^{4}$ is a conservative ${ }^{5}$ discretization which captures the essential physical transport property of supersonic flow, that advected quantities directly influence the solution only downstream. The method is formally first-order accurate, but is competitive with second-order upwind schemes. For subsonic flow, the second upwind method corresponds to the Scharfetter-Gummel discretization for strong electric fields; for weak electric fields the Scharfetter-Gummel discretization corresponds to central differences [7].

Equations (10)-(13) have the form

$$
\left[\begin{array}{c}
f_{n} \\
f_{v} \\
f_{T} \\
f_{\phi}
\end{array}\right]=\frac{d}{d x}\left[\begin{array}{l}
v g_{n} \\
v g_{v} \\
v g_{T} \\
0
\end{array}\right]+\left[\begin{array}{l}
0 \\
h_{v} \\
h_{T} \\
h_{\phi}
\end{array}\right]+\left[\begin{array}{c}
0 \\
s_{v} \\
s_{T} \\
s_{\phi}
\end{array}\right]=0
$$

where

$$
\begin{aligned}
& g_{n}=n \quad g_{v}=m n v \\
& g_{T}=\frac{5}{2} n T+\frac{1}{2} m n v^{2}-e n \phi \\
& h_{v}=-e n \frac{d \phi}{d x}+\frac{d}{d x}(n T) \\
& h_{T}=-\frac{d}{d x}\left(\kappa \frac{d T}{d x}\right) \quad h_{\phi}=\epsilon \frac{d^{2} x}{d x^{2}}
\end{aligned}
$$

and where the source terms $s_{v}, s_{T}$, and $s_{\phi}$ depend only on $n, v$, and $T$. In the second upwind method, the advection terms $d(v g) / d x$ in (14) are discretized using second upwind differences

$$
\frac{d}{d x}(v g)_{i} \approx v_{i+1} g_{R}-v_{i} g_{L}
$$

where

$$
\begin{aligned}
& g_{R}= \begin{cases}g_{i} & \left(v_{i+1}>0\right) \\
g_{i+1} & \left(v_{i+1}<0\right)\end{cases} \\
& g_{L}= \begin{cases}g_{i-1} & \left(v_{i}>0\right) \\
g_{i} & \left(v_{i}<0\right)\end{cases}
\end{aligned}
$$

and central differences are used for $h_{p}, h_{T}$, and $h_{\phi}$. Recall that the velocity $v_{i+1}$ is located at the midpoint of element $l_{i+1}$.

To linearize the discretized version of (10)-(13), I use Newton's method

$$
\begin{gathered}
{\left[\begin{array}{c}
\delta n \\
\delta v \\
\delta T \\
\delta \phi
\end{array}\right]=-\left[\begin{array}{l}
f_{n} \\
f_{v} \\
f_{T} \\
f_{\phi}
\end{array}\right]=-f} \\
{\left[\begin{array}{l}
n \\
v \\
T \\
\phi
\end{array}\right] \leftarrow\left[\begin{array}{l}
n \\
v \\
T \\
\phi
\end{array}\right]+\left[\begin{array}{c}
\delta n \\
\delta v \\
\delta T \\
\delta \phi
\end{array}\right]}
\end{gathered}
$$

${ }^{4}$ See, e.g., [6]

${ }^{5}$ The first-order upwind method is not conservative at points where the velocity changes sign. where $J$ is the Jacobian and $t$ is a damping factor [8] between 0 and 1 , chosen to insure that the norm of the residual $f$ decreases monotonically.

Physical Parameters: In silicon, the effective electron mass $m=0.26 m_{e}$ at $300 \mathrm{~K}$ and $0.24 m_{e}$ at $77 \mathrm{~K}$, where $m_{e}$ is the electron mass, $\epsilon=11.7$, and $n_{i}=1.45 \times 10^{10} \mathrm{~cm}^{-3}$ at $300 \mathrm{~K}$ and $2.84 \times 10^{-20} \mathrm{~cm}^{-3}$ at $77 \mathrm{~K}$. The best value of $\kappa_{0}$ appears to be $\kappa_{0}=0.4$, according to comparisons of hydrodynamic and Monte Carlo simulations of the ballistic diode at $300 \mathrm{~K}$ [9] (using only one species of electrons). Monte Carlo simulations of the ballistic diode at $77 \mathrm{~K}$ are needed to determine the dependence of $\kappa_{0}$ on $T_{0}, T$, etc.

I use the following models for $\mu_{n o}$ and $v_{s}$ [5], [10]:

$$
\begin{aligned}
& \mu_{n 0}\left(T_{0}, N_{i}\right)=\mu_{\min }+\frac{\Delta \mu}{\left(1+\left(N_{i} / N_{\text {ref }}\right)^{\alpha}\right)} \\
& \mu_{\min }=80 \mathrm{~cm}^{2} / \mathrm{V} \cdot \mathrm{s} \\
& \begin{cases}\left(300 \mathrm{~K} / T_{0}\right)^{0.45} & \left(T_{0} \geq 200 \mathrm{~K}\right) \\
(300 / 200)^{0.45}\left(200 \mathrm{~K} / T_{0}\right)^{0.15} & \left(T_{0}<200 \mathrm{~K}\right)\end{cases} \\
& \Delta \mu=\left(300 \mathrm{~K} / T_{0}\right)^{2} 1430 \frac{\mathrm{cm}^{2}}{\mathrm{~V} \cdot \mathrm{s}}-\mu_{\text {min }} \\
& N_{\text {ref }}=\left(T_{0} / 300 \mathrm{~K}\right)^{3.2} 1.12 \times 10^{17} \mathrm{~cm}^{-3} \\
& \alpha=0.72\left(T_{0} / 300 \mathrm{~K}\right)^{0.065}
\end{aligned}
$$

where $N_{i}=N_{D}+N_{A}$ is the total impurity concentration, and

$$
v_{s}\left(T_{0}\right)=\frac{2.4}{1+0.8 \exp \left(0.5 T_{0} / 300 \mathrm{~K}\right)} \times 10^{7} \mathrm{~cm} / \mathrm{s} .
$$

\section{COMPUTATIONS fOR SUbSONIC Flow}

For the subsonic computations, I take a diode consisting of a $0.1-\mu \mathrm{m}$ source, a $0.4-\mu \mathrm{m}$ channel, and a $0.1-\mu \mathrm{m}$ drain. In the $\mathrm{n}^{+}$region, the doping density $N=5 \times 10^{17} \mathrm{~cm}^{-3}$, while in the $\mathrm{n}$ region $N=2 \times 10^{15} \mathrm{~cm}^{-3}$. (I use a hyperbolic tangent fitting over $\pm 0.05 \mu \mathrm{m}$ at the junctions. ) The ambient device temperature $T_{0}=300 \mathrm{~K}=0.0259 \mathrm{eV}$. The saturation velocity $v_{s}=$ $1.03 \times 10^{7} \mathrm{~cm} / \mathrm{s}$. Note that $v_{s}<c\left(T_{0}\right)=1.3 \times 10^{7} \mathrm{~cm} / \mathrm{s}$.

To validate the second upwind method for subsonic problems, I performed simulations (with 120 grid intervals) of the ballistic diode at $V=1 \mathrm{~V}$ using the hydrodynamic model. The simulations are in good agreement with other hydrodynamic and Monte Carlo simulations of the same device [9], [11]-[13], although there are differences due to the different numerical methods and the different models for $\tau_{p}, \tau_{w}$, and $\kappa$.

Fig. 1 compares the temperature of the electron gas for the central difference and second upwind methods. Note that the temperature increases as the electrons flow from left to right across the channel. The maximum temperature is five times higher than ambient. Recall that the drift-diffusion model assumes the electron temperature $T=T_{0}$ throughout the device. Also note the slight cooling of the electron gas as the electrons enter the channel. This cooling results from the electrons having to overcome a small potential barrier at the junction (see the electric field in Fig. 2, computed using the second upwind method).

In Fig. 3, I compare the electron velocity for the central difference and second upwind methods. Note that $v>v_{s}$ through- 


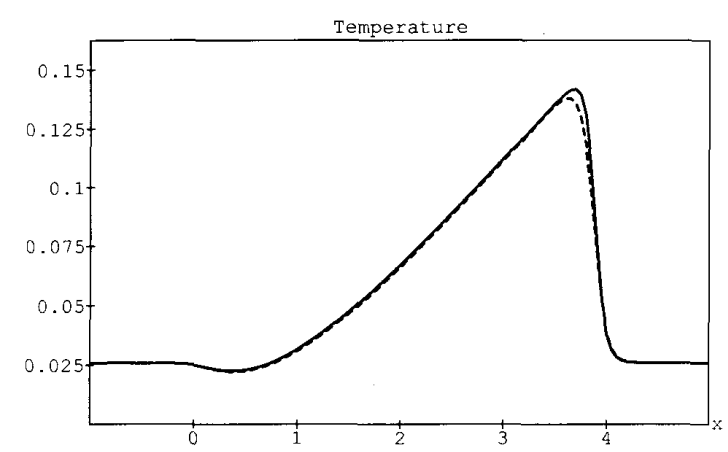

Fig. 1. Electron temperature in electron-volts for $V=1 \mathrm{~V}, 0.4-\mu \mathrm{m}$ channel, $300 \mathrm{~K}$ : central difference (solid) versus second upwind (dotted). $x$ is in $0.1 \mu \mathrm{m}$ for all figures.

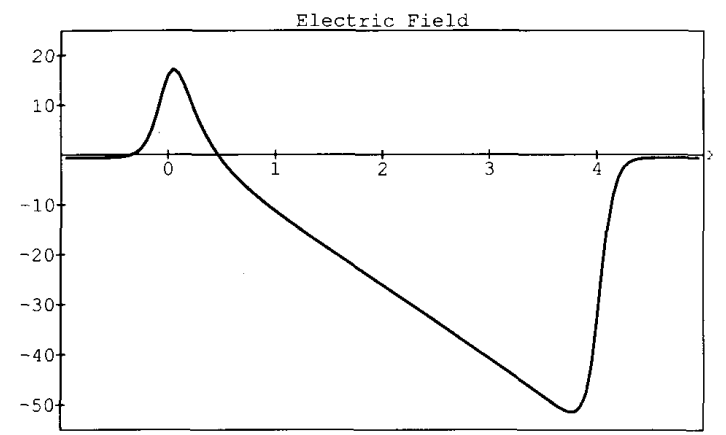

Fig. 2. Electric field in kilovolts per centimeter for $V=1 \mathrm{~V}, 0.4-\mu \mathrm{m}$ channel, $300 \mathrm{~K}$.

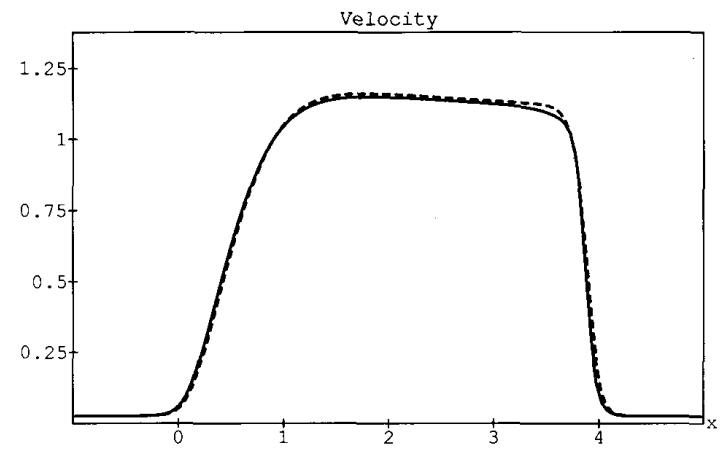

Fig. 3. Electron velocity in $10^{7} \mathrm{~cm} / \mathrm{s}$ for $V=1 \mathrm{~V}, 0.4-\mu \mathrm{m}$ channel $300 \mathrm{~K}$ : central difference (solid) versus second upwind (dotted).

out most of the channel. Typical Mach numbers for these flows are $\leqslant 0.7$. The difference $n-N$ between the electron density and the density of the background impurity ions is shown in Fig. 4 (using the second upwind method). Note the dipole domain (an electron depletion region followed by an accumulation region) at the source-channel junction and the large depletion region at the channel-drain junction. The effect of the dipole domain is to set up a local electric field opposing diffusion of electrons from the source to the channel. The large depletion region at the channel-drain junction provides electrons to the channel, and (with the channel) sets up the electric field driving electrons from the channel to the drain. Even for this $0.4-\mu \mathrm{m}$

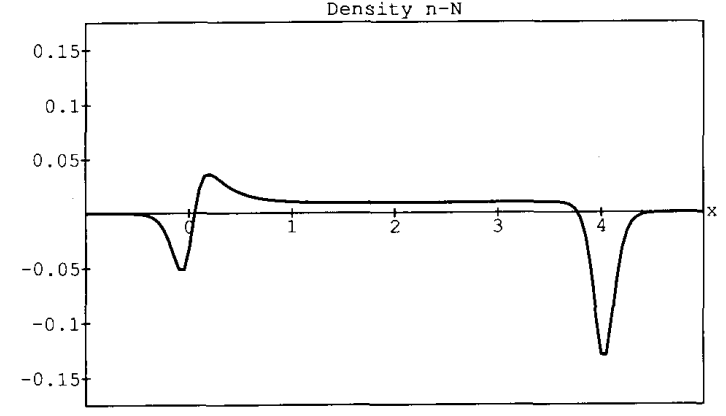

Fig. 4. $n-N$ in $10^{18} \mathrm{~cm}^{-3}$ for $V=1 \mathrm{~V}, 0.4-\mu \mathrm{m}$ channel, $300 \mathrm{~K}$.

device at a $1-\mathrm{V}$ bias, the electron kinetic energy can be as much as $15 \%$ of the total energy.

\section{Computations for Transonic Flow}

Higher electron velocities can be obtained by increasing the bias $V$ across a device or by making the active device length shorter. Since the electron soundspeed $c=\sqrt{T / m}$, higher electron Mach numbers may also be obtained by lowering the ambient temperature $T_{0}$.

Preliminary numerical evidence for a steady-state electron shock wave in the ballistic diode (at both 300 and $77 \mathrm{~K}$ ) using the hydrodynamic model was given by the author in [14]. The mathematical analogy with gas dynamics (including the mathematical necessity of shock waves in the hydrodynamic model) was made in [14] and by the author, Jerome, and Rose in [13]. Fatemi, Jerome, and Osher presented numerical evidence for the existence of a transient shock wave in the ballistic diode at $50 \mathrm{~K}$ using a high-order nonoscillatory upwind scheme in [15], although the models for $m, \tau_{p}, \tau_{w}$, and $\kappa$ were unphysical at 50 $\mathrm{K}$.

In this investigation, I will present two parameter regimes for the ballistic diode in which there is a transition from subsonic to supersonic electron flow just to the right of the $n^{+}-n$ junction and from supersonic to subsonic flow to the left of the $n-n^{+}$ junction. In analogy with gas dynamical flow in a Laval nozzle, ${ }^{6}$ a shock wave develops at the transition from supersonic to subsonic flow. The $n^{+}-n-n^{+}$doping of the ballistic diode corresponds to the converging-diverging geometry of the Laval nozzle.

The hydrodynamic shocks simulated below have a more complicated structure than shocks supported by the Euler equations of gas dynamics, due to the heat conduction term $\nabla \cdot(\kappa \nabla T)$ in (3), the relaxation time source terms in (2) and (3), and the coupling of the electron gas to the electric field. For the (inviscid) Euler equations, a shock wave is an exact discontinuity in $n, v$, and $T$, where $v$ is the normal velocity of the gas [1]. In gas dynamics with heat conduction and viscosity, a shock wave varies rapidly but smoothly over a short viscous length scale [1], [17], [18]. (The hydrodynamic model neglects the effects of viscosity in the electron gas.) If there is heat conduction, but no viscosity, the situation is more complex [17], [18]. In the frame of the shock, the flow ahead of the shock is supersonic and behind the shock is subsonic. If the Mach number $M$ of the flow ahead of the shock is greater than 1 but less than a critical

${ }^{6}$ See [16] for a theoretical and numerical investigation of a steady-state shock wave in a Laval nozzle. 


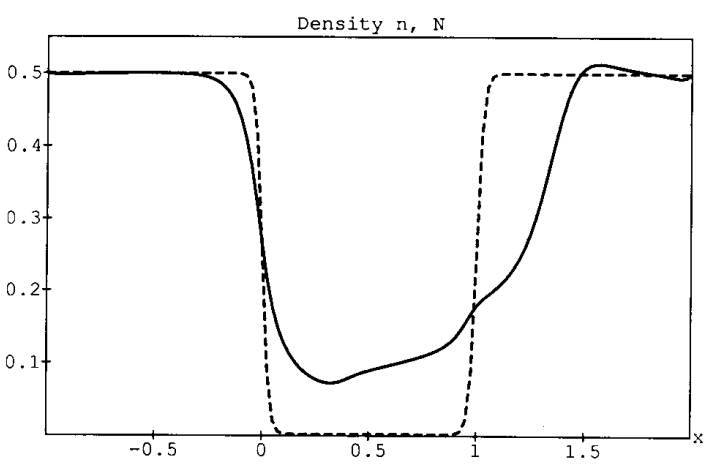

Fig. 5. Doping profile (dotted) and electron density (solid) in $10^{18} \mathrm{~cm}^{-3}$ at $V=1 \mathrm{~V}$ for $0.1-\mu \mathrm{m}$ channel device at $300 \mathrm{~K}$

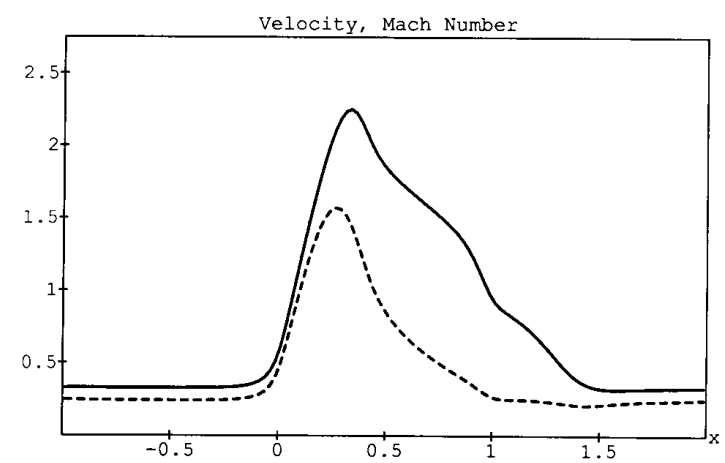

Fig. 6. Electron velocity (solid) in $10^{7} \mathrm{~cm} / \mathrm{s}$ and Mach number (dotted) for $V=1 \mathrm{~V}, 0.1-\mu \mathrm{m}$ channel, $300 \mathrm{~K}$.

Mach number $M_{c}$, the shock is a rapidly varying smooth profile. If $M>M_{c}$, then there is an outer profile to the shock wave with an inner exact discontinuity [17]. $\left(M_{c}=v_{c} / c \approx 1.74\right.$ for a polytropic gas with $\gamma=5 / 3$, where $c=\sqrt{T / m}$.) In simulations with numerical viscosity, the inner discontinuity will be spread out over several grid intervals.

The transonic computations use the second upwind method. (The central difference method becomes unstable just as the maximum Mach number of the flow slightly exceeds 1 .)

\section{A. Electron Shock Wave at $300 \mathrm{~K}$}

For the transonic computations at $300 \mathrm{~K}$, I take a diode consisting of a $0.1-\mu \mathrm{m}$ source, a $0.1-\mu \mathrm{m}$ channel, and a $0.1-\mu \mathrm{m}$ drain. In the $\mathrm{n}^{+}$region, the doping density $N=5 \times 10^{17} \mathrm{~cm}^{-3}$, while in the $\mathrm{n}$ region $N=2 \times 10^{15} \mathrm{~cm}^{-3}$ (see Fig. 5). (I use a hyperbolic tangent fitting over $\pm 0.0125 \mu \mathrm{m}$ at the junctions.)

Figs. 5-9 present a simulation of a Mach 1.6 electron shock wave at $V=1 \mathrm{~V}$ (with 120 grid intervals). Since the simulation is steady-state, we are in the rest frame of the shock. The shock profile develops at $x_{s} \approx 0.035 \mu \mathrm{m}$ (see Fig. 6), and is spread over about $5 \Delta x$. The electron flow is subsonic behind $(b)$ the shock profile and supersonic ahead $(a)$ of the shock profile. Note that the shock wave is a sharp profile in $v, M$, and $n$ (see Fig. 7), and that $n_{b}>n_{a}, v_{b}<v_{a}$.

The simulation of the $300 \mathrm{~K}$ shock wave has converged under mesh refinement with 120 grid intervals. The width of the shock wave (from the peak at $x \approx 0.033125 \mu \mathrm{m}$ to the slight "bend" at $x \approx 0.044125 \mu \mathrm{m}$ ) is $0.0200,0.01250$, and $0.010000 \mu \mathrm{m}$ for 60,120 , and 240 grid intervals, respectively.

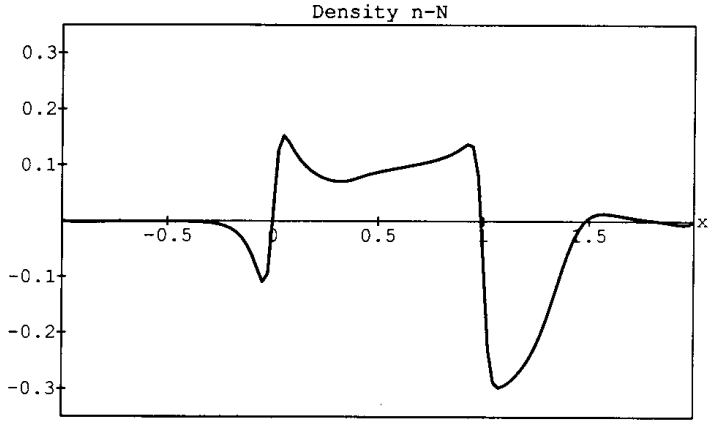

Fig. 7. $n-N$ in $10^{18} \mathrm{~cm}^{-3}$ for $V=1 \mathrm{~V}, 0.1-\mu \mathrm{m}$ channel, $300 \mathrm{~K}$.

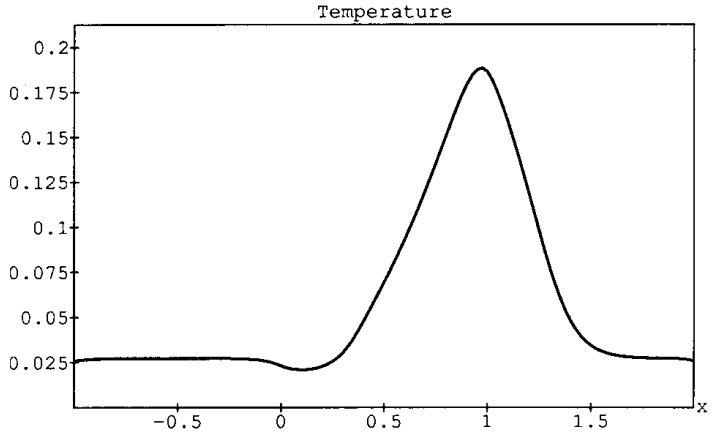

Fig. 8. Electron temperature in electron-volts for $V=1 \mathrm{~V}, 0.1-\mu \mathrm{m}$ channel, $300 \mathrm{~K}$.

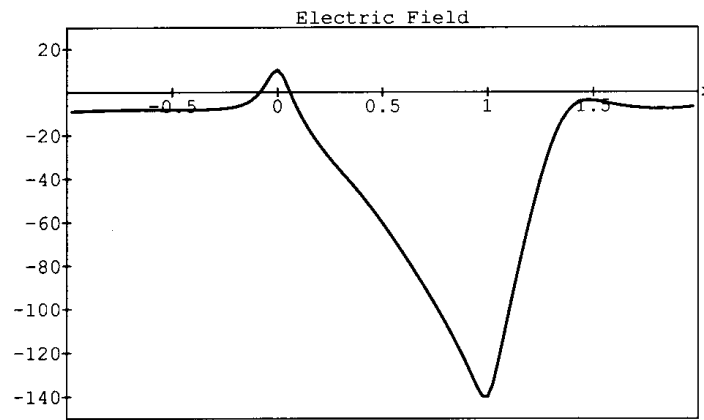

Fig. 9. Electric field in kilovolts per centimeter for $V=1 \mathrm{~V}, 0.1-\mu \mathrm{m}$ channel, $300 \mathrm{~K}$.

Fig. 5 shows the doping profile and the electron density. The electron density in the channel is almost two orders of magnitude greater than the background doping for this very short device. The dipole domain (Fig. 7) at the source-channel junction has been distorted into a new wave consisting of a depletion region followed by a region where the electron density is significantly greater than the background doping density. The large depletion region at the channel-drain junction extends well into the drain. The electron temperature (Fig. 8) is also elevated well into the drain. Note the slight cooling of the electron gas as the electrons enter the channel, due to the small potential barrier at the junction. The electric field in Fig. 9 is qualitatively similar to the field in Fig. 2, but the peak value is $3 \times$ higher. For this flow the electron kinetic energy can be as large as $45 \%$ of the total energy. 


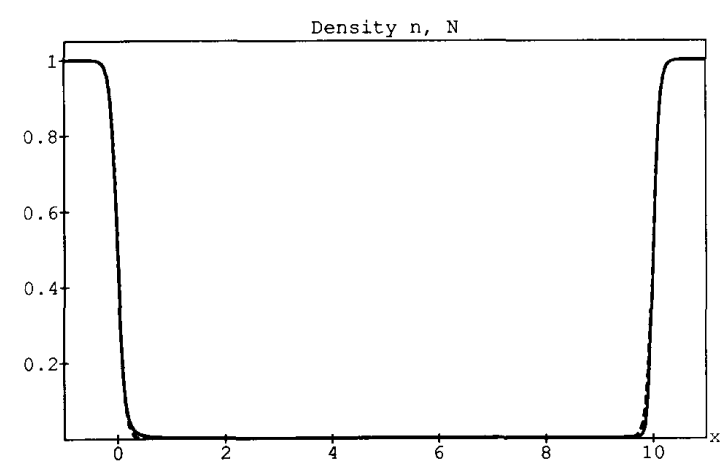

Fig. 10. Doping profile (dotted) and electron density (solid) in $10^{18} \mathrm{~cm}^{-3}$ at $V=1 \mathrm{~V}$ for $1-\mu \mathrm{m}$ channel device at $77 \mathrm{~K}$.

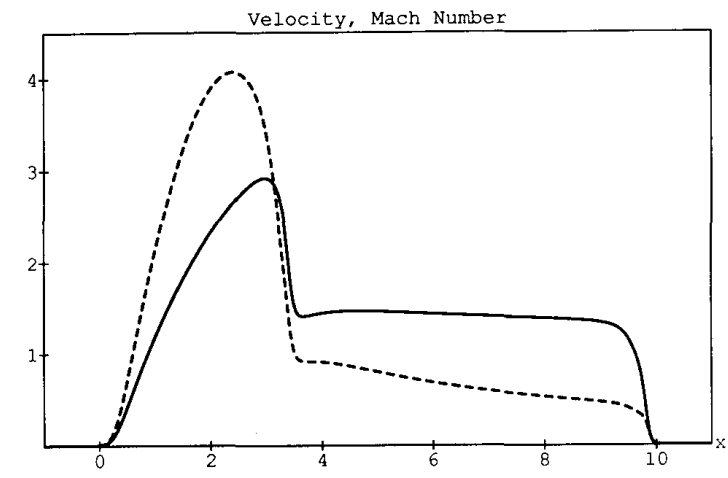

Fig. 11. Electron velocity (solid) in $10^{7} \mathrm{~cm} / \mathrm{s}$ and Mach number (dotted) for $V=1 \mathrm{~V}, 1-\mu \mathrm{m}$ channel, $77 \mathrm{~K}$.

\section{B. Electron Shock Wave at $77 \mathrm{~K}$}

For the transonic computations at $77 \mathrm{~K}$, I take a diode consisting of a $0.1-\mu \mathrm{m}$ source, a $1.0-\mu \mathrm{m}$ channel, and a $0.1-\mu \mathrm{m}$ drain. In the $\mathrm{n}^{+}$region, the doping density $N=10^{18} \mathrm{~cm}^{-3}$ at $77 \mathrm{~K}$, while in the n region $N=10^{15} \mathrm{~cm}^{-3}$ at $77 \mathrm{~K}$ (see Fig. 10 ). (I use a hyperbolic tangent fitting over $\pm 0.05 \mu \mathrm{m}$ at the junctions.) The ambient device temperature $T_{0}=77 \mathrm{~K}=$ $0.00665 \mathrm{eV}$. The saturation velocity $v_{s}=1.26 \times 10^{7} \mathrm{~cm} / \mathrm{s}$. Note that $v_{s}>c\left(T_{0}\right)=0.66 \times 10^{7} \mathrm{~cm} / \mathrm{s}$.

Figs. 10-14 present a simulation of a Mach 4.1 electron shock wave at $V=1 \mathrm{~V}$ (with 240 grid intervals). The shock profile develops at $x_{s} \approx 0.3 \mu \mathrm{m}$ (see Fig. 11), and is spread over about $10 \Delta x$. The electron flow is subsonic behind the shock profile and supersonic ahead of the shock profile. Note that the shock wave is a much sharper profile in $v, M$, and $n$ (see Fig. 12) than the Mach 1.6 shock wave, and that $n_{b}>n_{a}, v_{b}<v_{a}$.

The simulation of the $77 \mathrm{~K}$ shock wave has converged under mesh refinement with 240 grid intervals. The width of the shock wave (from the peak at $x \approx 0.30125 \mu \mathrm{m}$ to the right-angle "bend" at $x \approx 0.35125 \mu \mathrm{m}$ ) is $0.08,0.060,0.0550$, and $0.05000 \mu \mathrm{m}$ for $60,120,240$, and 480 grid intervals, respectively.

Fig. 10 shows the doping profile and the electron density. The dipole domain (Fig. 12) at the source-channel junction and the large depletion region at the channel-drain junction are clearly visible. The electron temperature is plotted in Fig. 13. Note the dramatic cooling of the electron gas as the electrons enter the channel, due to the potential barrier at the junction.

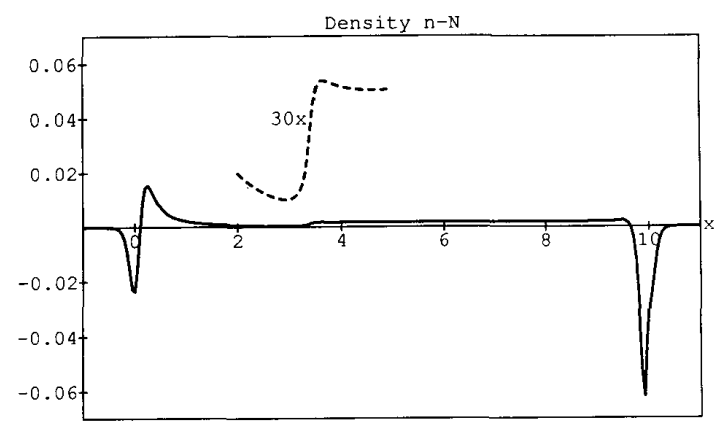

Fig. 12. $n-N$ in $10^{18} \mathrm{~cm}^{-3}$ for $V=1 \mathrm{~V}, 1-\mu \mathrm{m}$ channel, $77 \mathrm{~K}$. The dotted curve is $n-N$ magnified $30 \times$.

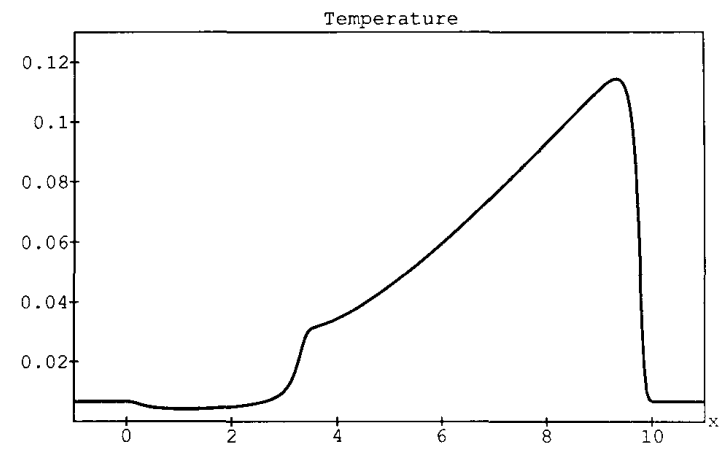

Fig. 13. Electron temperature in electron-volts for $V=1 \mathrm{~V}, 1-\mu \mathrm{m}$ channel, $77 \mathrm{~K}$.

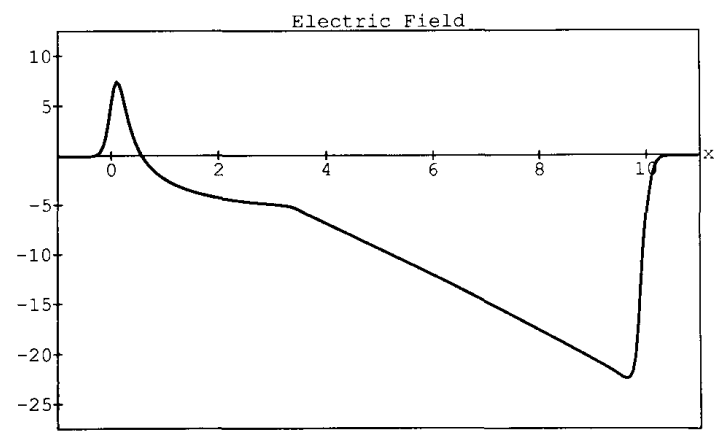

Fig. 14. Electric field in kilovolts per centimeter for $V=1 \mathrm{~V}, 1-\mu \mathrm{m}$ channel, $77 \mathrm{~K}$.

The electric field in Fig. 14 differs qualitatively from the fields in Figs. 2 and 9 in that the presence of the strong shock wave is manifest near $x=0.3 \mu \mathrm{m}$. The electron kinetic energy can be as large as $80 \%$ of the total energy for this flow.

\section{CONCLUSION}

Supersonic flow need not involve shock waves. The electron flow changes smoothly from subsonic to supersonic in Figs. 6 and 11 as the electrons enter the channel. It is the transition from supersonic flow to subsonic flow that generically necessitates a shock wave in gas dynamics-that is, a wave over which density, velocity, and (if heat conduction equals zero) temperature change very rapidly. The $\mathrm{n}^{+}$drain in the source- 
channel-drain structure of the ballistic diode provides the mechanism to force a supersonic flow in the channel back down to subsonic flow.

In an ideal limit (no viscosity and no heat conduction), the shock wave is an exact discontinuity in density, velocity, and temperature. Based on the analogy with gas dynamics, I expect the Mach 4.1 shock wave at $77 \mathrm{~K}$ to consist of an outer shock profile plus an inner exact discontinuity, which could be resolved computationally over 1-2 grid intervals with a higher order Godunov method. With the second upwind method, the inner discontinuity appears to be spread over $3 \Delta x$ with 480 grid intervals ( the electron velocity changes from $2.31 \times 10^{7}$ to 1.68 $\times 10^{7} \mathrm{~cm} / \mathrm{s}$ over $3 \Delta x$ ). Note that the discontinuous jump in $v$ (and $n$ ) for the $77 \mathrm{~K}$ shock waves implies a discontinuity in $d T / d x{ }^{7}$ which is visible in Fig. 13. (The Mach 1.6 shock wave at $300 \mathrm{~K}$ should be a smooth shock profile.)

Since the ballistic diode models the channel of a MOSFET, the development of the electron shock wave should have implications for MOSFET performance, perhaps as a threshold effect in the $I-V$ curves or switching time. Computations in progress for a two-dimensional FET should reveal more clearly further implications of the electron shock wave.

The 1D shock computations imply that the electron shock waves are an integral part of the hydrodynamic model. The shock waves allow for higher electron velocities in the diode channel (the maximum $v$ in Figs. 6 and 11 is greater than $2 v_{s}$ ) and thus faster switching times, and provide a richer spacecharge structure in the diode channel.

\section{REFERENCES}

[1] R. Courant and K. O. Friedrichs, Supersonic Flow and Shock Waves. New York, NY: Springer-Verlag, 1948.

[2] K. Blфtekjær, "Transport equations for electrons in two-valley semiconductors," IEEE Trans. Electron Devices, vol. ED-17, pp. 38-47, 1970.

[3] P. D. Lax, Hyperbolic Systems of Conservation Laws and the Mathematical Theory of Shock Waves. Philadelphia, PA: SIAM, 1972.

[4] C. L. Gardner, "Nonlinear hyperbolic waves in the hydrodynamic model for semiconductor devices," to appear.

[5] G. Baccarani and M. R. Wordeman, "An investigation of steadystate velocity overshoot effects in $\mathrm{Si}$ and GaAs devices," SolidState Electron., vol. 28, pp. 407-416, 1985.

${ }^{7}$ Integrating (12) over a wave yields $1 / 2 m n v\left[v^{2}\right]=\kappa[d T / d x]$, where $[\psi]$ indicates the jump $\psi_{+}-\psi_{-}$across the wave [4].
[6] P. J. Roache, Computational Fluid Dynamics. Albuquerque, NM: Hermosa, 1982

[7] J. P. Kreskovsky, "A hybrid central difference scheme for solidstate device simulation," IEEE Trans. Electron Devices, vol. ED-34, pp. 1128-1133, 1987

[8] R. E. Bank and D. J. Rose, "Global approximate Newton methods," Numerische Mathematik, vol. 37, pp. 279-295, 1981.

[9] A. Gnudi, F. Odeh, and M. Rudan, "Investigation of non-local transport phenomena in small semiconductor devices," to appear.

[10] S. Shelberherr, "MOS device modeling at $77 \mathrm{~K}$," IEEE Trans. Electron Devices, vol. 36, pp. 1464-1474, 1989.

[11] F. Odeh, M. Rudan, and J. White, "Numerical solution of the hydrodynamic model for a one-dimensional semiconductor device," COMPEL, vol. 6, pp. 151-170, 1987.

[12] M. V. Fischetti and M. Rudan, "Hýdrodynamic and Monte Carlo simulation of an $\mathrm{n}^{+}-\mathrm{n}^{-} \mathrm{n}^{+}$submicron device," IEEE Trans. Electron Devices, to appear.

[13] C. L. Gardner, J. W. Jerome, and D. J. Rose, "Numerical meth ods for the hydrodynamic device model: Subsonic flow," IEEE Trans. Computer-Aided Des., vol. 8, pp. 501-507, 1989.

[14] C. L. Gardner, "Nonlinear hyperbolic waves in the hydrodynamic model for semiconductor devices," presented at VLSI Minisymposium, SIAM Annual Meeting, Minneapolis, MN, July $15,1988$.

[15] E. Fatemi, J. W. Jerome, and S. Osher, "Solution of the hydrodynamic device model using high-order non-oscillatory shock capturing algorithms," IEEE Trans. Computer-Aided Des., to appear.

[16] J. Glimm, G. Marshall, and B. Plohr, "A generalized Riemann problem for quasi-one-dimensional gas flows," Adv. Appl. Math. vol. 5, pp. 1-30, 1984

[17] Ya. B. Zel'dovich and Yu. P. Raizer, Physics of Shock Waves and High-Temperature Hydrodynamic Phenomena. New York, NY: Academic Press, 1966.

[18] R. Menikoff and B. J. Plohr, "The Riemann problem for fluid flow of real materials," Rev. Mod. Phys., vol. 61, pp. 75-130, 1989

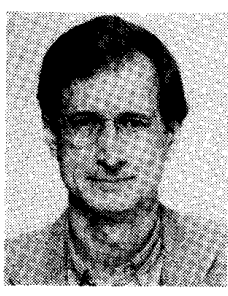

Carl L. Gardner received the B.A. degree in mathematics from Duke University, Durham, $\mathrm{NC}$ in 1973, and the Ph.D. degree in physics from MIT, Cambridge, MA, in 1981.

$\mathrm{He}$ is currently an Associate Professor of Computer Science at Duke University. Previously he was an Assistant Professor of Computer Science at Duke University, an Associate Research Scientist at the Courant Institute, Assistant Professor of Physics at Bowdoin College, and Instructor of Physics at MIT. His current research interests lie in semiconductor device and process simulations, and computational granular and fluid dynamics.

Dr. Gardner is a member of SIAM and the American Physical Society. 